\title{
Structured Firewall Design
}

\author{
Mohamed G. Gouda and Alex X. Liu* \\ Department of Computer Sciences \\ The University of Texas at Austin \\ Austin, Texas 78712-0233, U.S.A. \\ \{gouda, alex\}@cs.utexas.edu
}

\begin{abstract}
A firewall is a security guard placed at the point of entry between a private network and the outside Internet such that all incoming and outgoing packets have to pass through it. The function of a firewall is to examine every incoming or outgoing packet and decide whether to accept or discard it. This function is conventionally specified by a sequence of rules, where rules often conflict. To resolve conflicts, the decision for each packet is the decision of the first rule that the packet matches. The current practice of designing a firewall directly as a sequence of rules suffers from three types of major problems: (1) the consistency problem, which means that it is difficult to order the rules correctly; (2) the completeness problem, which means that it is difficult to ensure thorough consideration for all types of traffic; (3) the compactness problem, which means that it is difficult to keep the number of rules small (because some rules may be redundant and some rules may be combined into one rule).

To achieve consistency, completeness, and compactness, we propose a new method called Structured Firewall Design, which consists of two steps. First, one designs a firewall using a Firewall Decision Diagram instead of a sequence of often conflicting rules. Second, a program converts the firewall decision diagram into a compact, yet functionally equivalent, sequence of rules. This method addresses the consistency problem because a firewall decision diagram is conflict-free. It addresses the completeness problem because the syntactic requirements of a firewall decision diagram force the designer to consider all types of traffic. It also addresses the compactness problem because in the second step we use two algorithms (namely FDD reduction and FDD marking) to combine rules together, and one algorithm (namely Firewall compaction) to remove redundant rules. Moreover, the techniques and algorithms presented in this paper are extensible to other rule-based systems such as IPsec rules.
\end{abstract}

Keywords: Network security; Firewall; Firewall design; Firewall policy

\section{Introduction}

\section{$1.1 \quad$ Firewall Basics}

Firewalls are crucial elements in network security, and have been widely deployed in most businesses and institutions for securing private networks. A firewall is placed at the point of entry between a private network and the outside Internet

\footnotetext{
* Alex X. Liu is the corresponding author of this paper. Email: alex@cs.utexas.edu.
} Telephone: +1 (512) 471-9711. Fax: +1 (512) 471-9536 
such that all incoming and outgoing packets have to pass through it. The function of a firewall is to examine every incoming or outgoing packet and decide whether to accept or discard it. A packet can be viewed as a tuple with a finite number of fields such as source IP address, destination IP address, source port number, destination port number, and protocol type. The function of a firewall is conventionally specified as a sequence of rules. Each rule in a firewall is of the form

$$
\langle\text { predicate }\rangle \rightarrow\langle\text { decision }\rangle
$$

The $\langle$ predicate $\rangle$ of a rule is a boolean expression over some packet fields together with the physical network interface on which a packet arrives. For simplicity, we assume that each packet has a field containing the identification of the network interface on which a packet arrives. The 〈decision $\rangle$ of a rule can be accept, or discard, or a combination of these decisions with other options such as a logging option. For simplicity, we assume that the $\langle$ decision $\rangle$ of a rule is either accept or discard.

A packet matches a rule if and only if (iff) the packet satisfies the predicate of the rule. The rules in a firewall often conflict. Two rules in a firewall conflict iff they overlap and also have different decisions. Two rules in a firewall overlap iff there is at least one packet that can match both rules. Due to conflicts among rules, a packet may match more than one rule in a firewall, and the rules that a packet matches may have different decisions. To resolve conflicts, the decision for each packet is the decision of the first (i.e., highest priority) rule that the packet matches. Consequently, the rules in a firewall are order sensitive. To ensure that every packet has at least one matching rule in a firewall, the predicate of the last rule in a firewall is usually a tautology. The last rule of a firewall is usually called the default rule of the firewall.

\subsection{Consistency, Completeness and Compactness}

Because of the conflicts and order sensitivity of firewall rules, designing a firewall directly as a sequence of rules suffers from these three problems: the consistency problem, the completeness problem, and the compactness problem. Next, we expatiate on these three problems via a simple firewall example shown in Figure 1. This firewall resides on a gateway router that connects a private network to the outside Internet. The gateway router has two interfaces: interface 0 , which connects the router to the outside Internet, and interface 1, which connects the router to the private network. In this example, we assume that every packet has the following five fields.

\begin{tabular}{|l|l|}
\hline name & meaning \\
\hline $\mathrm{I}$ & Interface \\
\hline $\mathrm{S}$ & Source IP address \\
\hline $\mathrm{D}$ & Destination IP address \\
\hline $\mathrm{N}$ & Destination Port Number \\
\hline $\mathrm{P}$ & Protocol Type \\
\hline
\end{tabular}

A firewall on the Internet typically consists of hundreds or thousands of rules. Here for simplicity, this firewall example only has four rules. Although this firewall is small, it exemplifies all the following three problems.

1. Consistency Problem: It is difficult to order the rules in a firewall correctly. This difficulty mainly comes from conflicts among rules. Because rules 


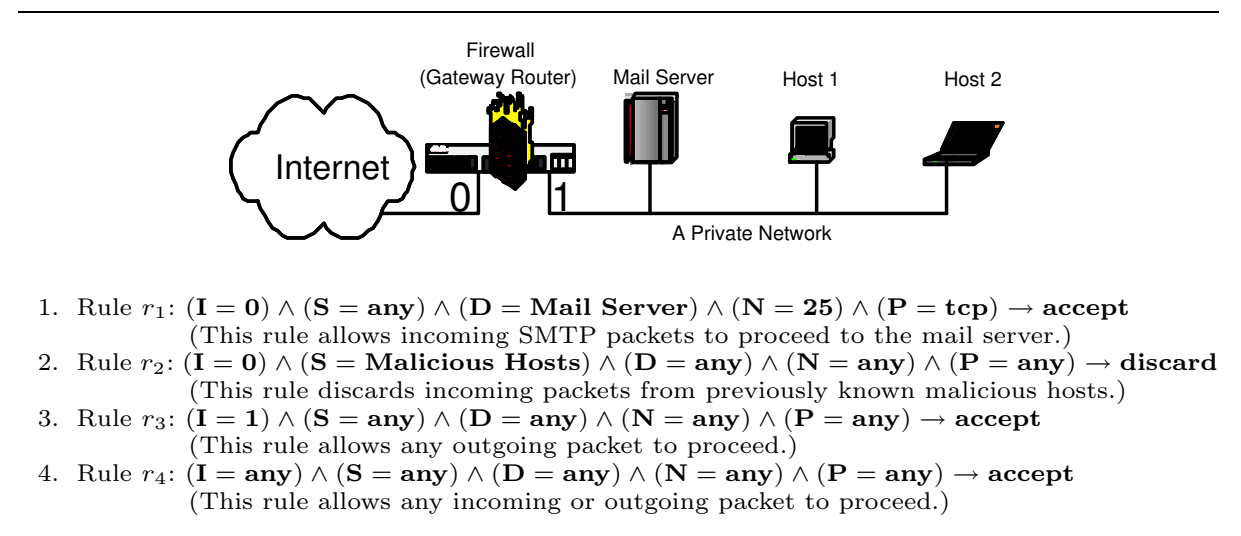

Figure 1. A Firewall Example

often conflict, the order of the rules in a firewall is critical. The decision for every packet is the decision of the first rule that the packet matches. In the firewall example in Figure 1, rule $r_{1}$ and $r_{2}$ conflict since the SMTP packets from previously known malicious hosts to the mail server match both rules and the decisions of $r_{1}$ and $r_{2}$ are different. Because $r_{1}$ is listed before $r_{2}$ and the decision of rule $r_{1}$ is "accept", the SMTP packets from previously known malicious hosts are allowed to proceed to the mail server. However, such packets probably should be prohibited from reaching the mail server because they originate from malicious hosts. Therefore, rules $r_{1}$ and $r_{2}$ probably should be swapped.

Because of the conflicts, the net effect of a rule cannot be understood by the literal meaning of the rule. The decision of a rule affects the fate of the packets that match this rule but does not match any rule listed before this rule. To understand one single rule $r_{i}$, one needs to go through all the rules from $r_{1}$ to $r_{i-1}$, and for every rule $r_{j}$, where $1 \leq j \leq i-1$, one needs to figure out the logical relationship between the predicate of $r_{j}$ and that of $r_{i}$. In the firewall example in Figure 1, the net effect of rule $r_{2}$ is not to "discard all packets originated from previously known malicious hosts", but rather is to "discard all non-SMTP packets originated from previously known malicious hosts". The difficulty in understanding firewall rules in turn makes the design and maintenance of a firewall error-prone. Maintenance of a firewall usually involves inserting, deleting or updating rules, and reporting the function of the firewall to others such as managers. All of these tasks require precise understanding of firewalls, which is difficult, especially when the firewall administrator is forced to maintain a legacy firewall that is not originally designed by him.

2. Completeness Problem: It is difficult to ensure that all possible packets are considered. To ensure that every packet has at least one matching rule in a firewall, the common practice is to make the predicate of the last rule a tautology. This is clearly not a good way to ensure the thorough consideration of all possible packets. In the firewall example in Figure 1, due to the last rule $r_{4}$, non-email packets from the outside to the mail server and 
email packets from the outside to the hosts other than the mail server are accepted by the firewall. However, these two types of traffic probably should be blocked. A mail server is usually dedicated to email service only. When a host other than the mail server starts to behave like a mail server, it could an indication that the host has been hacked and it is sending out spam. To block these two types of traffic, the following two rules should be inserted immediately after rule $r_{1}$ in the above firewall:
(a) $(I=0) \wedge(S=$ any $) \wedge(D=$ Mail Server $) \wedge(N=$ any $) \wedge(P=$ any $) \rightarrow$ discard
(b) $(I=0) \wedge(S=$ any $) \wedge(D=$ any $) \wedge(N=25) \wedge(P=t c p) \rightarrow$ discard

3. Compactness Problem: A poorly designed firewall often has redundant rules. A rule in a firewall is redundant iff removing the rule does not change the function of the firewall, i.e., does not change the decision of the firewall for every packet. In the above firewall example in Figure 1, rule $r_{3}$ is redundant. This is because all the packets that match $r_{3}$ but do not match $r_{1}$ and $r_{2}$ also match $r_{4}$, and both $r_{3}$ and $r_{4}$ have the same decision. Therefore, this firewall can be made more compact by removing rule $r_{3}$.

The consistency problem and the completeness problem cause firewall errors. An error in a firewall means that the firewall either accepts some malicious packets, which consequently creates security holes on the firewall, or discards some legitimate packets, which consequently disrupts normal businesses. Given the importance of firewalls, such errors are not acceptable. Unfortunately, it has been observed that most firewalls on the Internet are poorly designed and have many errors in their rules [27].

The compactness problem causes low firewall performance. In general, the smaller the number of rules that a firewall has, the faster the firewall can map a packet to the decision of the first rule the packet matches. Reducing the number of rules is especially useful for the firewalls that use TCAM (Ternary Content Addressable Memory). Such firewalls use $O(n)$ space (where $n$ is the number of rules) and constant time in mapping a packet to a decision. Despite the high performance of such TCAM-based firewalls, TCAM has very limited size and consumes much more power as the number of rules increases. Size limitation and power consumption are the two major issues for TCAM-based firewalls.

\subsection{Structured Firewall Design}

To achieve consistency, completeness, and compactness, we propose a new method called Structured Firewall Design, which consists of two steps. First, one designs a firewall using a Firewall Decision Diagram (FDD for short) instead of a sequence of often conflicting rules. Second, a program converts the FDD into a compact, yet functionally equivalent, sequence of rules. This method addresses the consistency problem because an FDD is conflict-free. It addresses the completeness problem because the syntactic requirements of an FDD force the designer to consider all types of traffic. It also addresses the compactness problem because in the second step we use two algorithms (namely FDD reduction and FDD marking) to combine rules together, and one algorithm (namely Firewall compaction) to remove redundant rules.

In some sense, our method of structured firewall design is like the method of structured programming, and the method of designing a firewall directly as 
a sequence of conflicting rules is like the method of writing a program with many goto statements. In late 1960s, Dijkstra pointed out that goto statements are considered harmful [10] because a program with many goto statements is very difficult to understand and therefore writing such a program is very error prone. Similarly, a firewall of a sequence of conflicting rules is very difficult to understand and writing a sequence of conflicting rules directly is extremely error prone.

Using the method of structured firewall design, the firewall administrator only deals with the FDD that uniquely represents the semantics of a firewall. The FDD is essentially the formal specification of a firewall. Since an FDD can be converted to an equivalent sequence of rules, our method does not require any modification to any existing firewall, which takes a sequence of rules as its configuration. Whenever the firewall administrator wants to change the function of his firewall, he only needs to modify the FDD and then use programs to automatically generate a new sequence of rules. This process is like a programmer first modifies his source code and then compiles it again.

Note that this paper is primarily on how to design stateless firewalls. There are two types of firewalls: stateless firewalls and stateful firewalls. If a firewall decides the fate of every packet solely by examining the packet itself, then the firewall is called a stateless firewall. If a firewall decides the fate of some packet not only by examining the packet itself but also by examining the packets that the firewall has accepted previously, then the firewall is called a stateful firewall. Studying how to design stateless firewall is particularly important for two major reasons. First, many stateless firewall products such as ipchains [2] have been widely deployed. Second, most of the rules in a stateful firewall are stateless rules [13]. Studying the design of stateless firewalls is the foundation for further exploration of the design of stateful firewalls. Although this paper concerns about firewall design, the techniques and algorithms presented in this paper are extensible to other rule-based systems [23].

The rest of this paper proceeds as follows. In Section 2, we examine related work. In Section 3, we introduce Firewall Decision Diagrams. In Section 4, we present Algorithm 1 whose function is to reduce the size of a user specified FDD. In Section 5, we present Algorithm 2 whose function is to do some marking on the reduced FDD. In Section 6, we present Algorithm 3 whose function is to generate firewall rules with the help of the marking information produced by Algorithm 2. In Section 7, we present Algorithm 4 whose function is to remove redundant rules from the firewall rules generated by Algorithm 3. In Section 8, we present Algorithm 5 whose function is to simplify firewall rules. In Section 10 , we give concluding remarks.

\section{Related Work}

It has been observed that most firewalls on the Internet are poorly designed and have many configuration errors in their rules $[9,27]$. There are two approaches to reduce firewall design errors. The first approach is to prevent errors from happening when designing firewalls. The second approach is to detect errors after firewalls have been designed.

With the first approach, people have tried to invent high-level languages that can be used to specify firewall rules. Examples of such languages are the simple model definition language in $[5,6]$, the Lisp-like language in [15], the declarative predicate language in [7], and the high level firewall language in [1]. These 
high-level firewall languages are helpful for designing firewalls because otherwise people have to use vendor specific languages to describe firewall rules. However, a firewall specified using these high-level firewall languages is still a sequence of rules and the rules may still conflict. The three problems of consistency, completeness and compactness that are inherent in designing a firewall by a sequence of rules still remain. Our firewall design method belongs to the first approach, but we propose a new firewall design paradigm that addresses the three problems of consistency, completeness and compactness. Our method is a complementary and prior step to those high-level firewall languages.

With the second approach, two methods have been tried previously. The first method is to analyze the function of a firewall by queries $[17,18,20,21,26]$. The basic idea is to issue queries such as "Which computers in the private network can receive packets from a known malicious host in the outside Internet?" This method is definitely helpful in detecting some of the errors in a firewall; however, this method is not guaranteed to detect all the errors in a firewall. The second method is conflict detection $[3,4,11,16,22]$. The basic idea of this method is to first detect all pairs of rules that conflict, and then the firewall designer manually examines every pair of conflicting rules to see whether the two rules need to be swapped or a new rule needs to be added. This manual checking is unreliable because the two conflicting rules have to be understood in the context of all the other rules in the firewall where the order of the other rules may be wrong. Since the number of conflicts in a firewall can be huge, this manual checking for each conflict could be tremendous work for the firewall designer.

Firewall vulnerabilities are discussed and classified in [12,19]. However, the focus of $[12,19]$ are the vulnerabilities of the packet filtering software and the supporting hardware part of a firewall, not the configuration of a firewall.

\section{Firewall Decision Diagrams}

A field $F_{i}$ is a variable whose domain, denoted $D\left(F_{i}\right)$, is a finite interval of nonnegative integers. For example, the domain of the source address in an IP packet is $\left[0,2^{32}-1\right]$.

A packet over fields $F_{1}, \cdots, F_{d}$ is a $d$-tuple $\left(p_{1}, \cdots, p_{d}\right)$ where each $p_{i}(1 \leq i \leq$ $d$ ) is an element of $D\left(F_{i}\right)$. We use $\Sigma$ to denote the set of all packets over fields $F_{1}, \cdots, F_{d}$. It follows that $\Sigma$ is a finite set and $|\Sigma|=\left|D\left(F_{1}\right)\right| \times \cdots \times\left|D\left(F_{d}\right)\right|$, where $|\Sigma|$ denotes the number of elements in set $\Sigma$ and each $\left|D\left(F_{i}\right)\right|(1 \leq i \leq d)$ denotes the number of elements in set $D\left(F_{i}\right)$.

A Firewall Decision Diagram (FDD) $f$ over fields $F_{1}, \cdots, F_{d}$ is an acyclic and directed graph that has the following five properties:

1. There is exactly one node in $f$ that has no incoming edges. This node is called the root of $f$. The nodes in $f$ that have no outgoing edges are called terminal nodes of $f$.

2. Each node $v$ in $f$ is labeled with a field, denoted $F(v)$, such that

$$
F(v) \in \begin{cases}\left\{F_{1}, \cdots, F_{d}\right\} & \text { if } v \text { is nonterminal } \\ \{\text { accept }, \text { discard }\} & \text { if } v \text { is terminal. }\end{cases}
$$

3. Each edge $e$ in $f$ is labeled with a nonempty set of integers, denoted $I(e)$, such that if $e$ is an outgoing edge of node $v$, then we have

$$
I(e) \subseteq D(F(v)) .
$$


4. A directed path in $f$ from the root to a terminal node is called a decision path. No two nodes on a decision path have the same label.

5 . The set of all outgoing edges of a node $v$ in $f$, denoted $E(v)$, satisfies the following two conditions:

(a) Consistency: $I(e) \cap I\left(e^{\prime}\right)=\emptyset$ for any two distinct edges $e$ and $e^{\prime}$ in $E(v)$.

(b) Completeness: $\bigcup_{e \in E(v)} I(e)=D(F(v))$.

Figure 2 shows an example of an FDD over two fields $F_{1}$ and $F_{2}$. The domain of each field is the interval $[1,10]$. Note that in labelling the terminal nodes, we use letter " $a$ " as a shorthand for "accept" and letter " $d$ " as a shorthand for "discard". These two notations are carried through the rest of this paper.

In this paper, the label of an edge in an FDD is always represented by the minimum number of non-overlapping integer intervals whose union equals the label of the edge. For example, one outgoing edge of the root is labeled with the set $\{1,2,3,4,9,10\}$, which is represented by the two intervals $[1,4]$ and $[9,10]$.

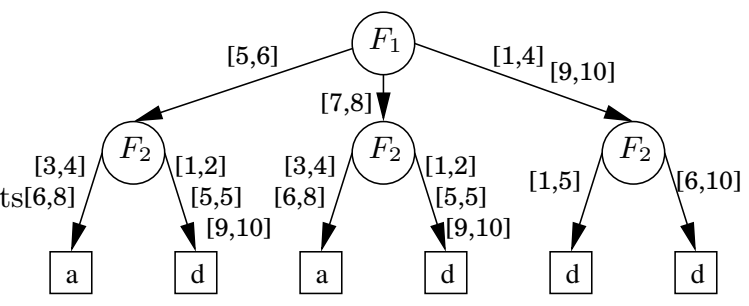

Figure 2. An FDD example

For brevity, in the rest of this paper, we assume that all packets and all FDDs are over the $d$ fields $F_{1}, \cdots, F_{d}$ unless otherwise specified.

A firewall decision diagram maps each packet to a decision by testing the packet down the diagram from the root to a terminal node, which indicates the decision of the firewall for the packet. Each nonterminal node in a firewall decision diagram specifies a test of a packet field, and each edge descending from that node corresponds to some possible values of that field. Each packet is mapped to a decision by starting at the root, testing the field that labels this node, then moving down the edge whose label contains the value of the packet field; this process is then repeated for the sub-diagram rooted at the new node.

A decision path in an FDD is represented by $\left\langle v_{1} e_{1} \cdots v_{k} e_{k} v_{k+1}\right\rangle$ where $v_{1}$ is the root, $v_{k+1}$ is a terminal node, and each $e_{i}(1 \leq i \leq k)$ is a directed edge from node $v_{i}$ to node $v_{i+1}$.

A decision path $\left\langle v_{1} e_{1} \cdots v_{k} e_{k} v_{k+1}\right\rangle$ in an FDD represents the following rule:

$$
F_{1} \in S_{1} \wedge \cdots \wedge F_{d} \in S_{d} \rightarrow\langle\text { decision }\rangle
$$

where

$S_{i}=\left\{\begin{array}{l}I\left(e_{j}\right) \text { if there is a node } v_{j} \text { in the decision path that is labeled with field } F_{i} \\ D\left(F_{i}\right) \text { otherwise }\end{array}\right.$ 
and $\langle$ decision $\rangle$ is the label of the terminal node $v_{k+1}$ in the path.

For an FDD $f$, we use $f$.rules to denote the set of all rules that are represented by all the decision paths of $f$. For any packet $p$, there is one and only one rule in f.rules that $p$ matches because of the consistency and completeness properties of an FDD. For example, the rules represented by all the decision paths of the FDD in Figure 2 are listed in Figure 3. Taking the example of the packet $(7,9)$, it matches only rule $r_{4}$ in Figure 3.

$\begin{array}{ll}r_{1}: F_{1} \in[5,6] \wedge F_{2} \in[3,4] \cup[6,8] & \rightarrow a \\ r_{2}: F_{1} \in[5,6] \wedge F_{2} \in[1,2] \cup[5,5] \cup[9,10] & \rightarrow d \\ r_{3}: F_{1} \in[7,8] \wedge F_{2} \in[3,4] \cup[6,8] & \rightarrow a \\ r_{4}: F_{1} \in[7,8] \wedge F_{2} \in[1,2] \cup[5,5] \cup[9,10] & \rightarrow d \\ r_{5}: F_{1} \in[1,4] \cup[9,10] \wedge F_{2} \in[1,5] & \rightarrow d \\ r_{6}: F_{1} \in[1,4] \cup[9,10] \wedge F_{2} \in[6,10] & \rightarrow d\end{array}$

Figure 3. All rules represented by FDD in Figure 2

The semantics of an FDD $f$ is defined as follows: for any packet $p, f$ maps $p$ to the decision of the rule (in fact the only rule) that $p$ matches in f.rules. More precisely, a packet $\left(p_{1}, \cdots, p_{d}\right)$ is accepted by an FDD $f$ iff there is a rule of the form

$$
F_{1} \in S_{1} \wedge \cdots \wedge F_{d} \in S_{d} \rightarrow \text { accept }
$$

in f.rules such that the condition $p_{1} \in S_{1} \wedge \cdots \wedge p_{d} \in S_{d}$ holds. Similarly, a packet $\left(p_{1}, \cdots, p_{d}\right)$ is discarded by an FDD $f$ iff there is a rule of the form

$$
F_{1} \in S_{1} \wedge \cdots \wedge F_{d} \in S_{d} \rightarrow \text { discard }
$$

in $f$.rules such that the condition $p_{1} \in S_{1} \wedge \cdots \wedge p_{d} \in S_{d}$ holds. For example, the packet $(6,8)$ is discarded by the FDD in Figure 2 because the rule that this packet matches is rule $r_{4}$ in Figure 3 and the decision of this rule is "discard".

Let $f$ be an FDD. The accept set of $f$, denoted f.accept, is the set of all packets that are accepted by $f$. Similarly, the discard set of $f$, denoted $f$.discard, is the set of all packets that are discarded by $f$. These two sets associated with an FDD precisely define the semantics of the FDD.

Based on the definitions of accept set and discard set, we have the following theorem. (Recall that $\Sigma$ denotes the set of all packets over the fields $F_{1}, \cdots, F_{d}$.)

Theorem 1 (Theorem of FDDs) For any FDD $f$, the following two conditions hold:

1. f.accept $\cap$ f.discard $=\emptyset$, and

2. f.accept $\cup$ f.discard $=\Sigma$

Two FDDs $f$ and $f^{\prime}$ are equivalent iff they have identical accept sets and identical discard sets, i.e., f.accept $=f^{\prime}$.accept and $f$.discard $=f^{\prime}$.discard.

There are some similarities between the structure of Firewall Decision Diagrams and that of Interval Decision Diagrams [25], which are mainly used in formal verification. However, there are two major differences. First, in a firewall 
decision diagram, the label of a nonterminal node must have a finite domain; while in an interval decision diagram, the label of a nonterminal node may have an infinite domain. Second, in a firewall decision diagram, the label of an edge is a set of integers which could be the union of several noncontinuous intervals; while in an interval decision diagram, the label of an edge is limited to only one interval. In broader sense, the structure of Firewall Decision Diagrams is also similar to other types of decision diagrams such as the Binary Decision Diagrams [8] and Decision Trees [24]. But note that the optimization goal of reducing the total number of simple rules generated is unique to firewall decision diagrams, which will be explored next.

\section{FDD Reduction}

In this section, we present an algorithm for reducing the number of decision paths in an FDD. This reduction helps to reduce the number of rules generated from an FDD. First, we introduce two concepts: isomorphic nodes in an FDD and reduced FDDs.

Two nodes $v$ and $v^{\prime}$ in an FDD are isomorphic iff $v$ and $v^{\prime}$ satisfy one of the following two conditions:

1. Both $v$ and $v^{\prime}$ are terminal nodes with identical labels.

2. Both $v$ and $v^{\prime}$ are nonterminal nodes and there is a one-to-one correspondence between the outgoing edges of $v$ and the outgoing edges of $v^{\prime}$ such that every pair of corresponding edges have identical labels and they both point to the same node.

An FDD $f$ is reduced iff it satisfies all of the following three conditions:

1. No node in $f$ has only one outgoing edge.

2. No two nodes in $f$ are isomorphic.

3. No two nodes have more than one edge between them.

Algorithm 1 (FDD reduction) in Figure 4 takes any FDD and outputs an equivalent but reduced FDD. The correctness of this algorithm follows directly from the semantics of FDDs. Note that this algorithm for reducing an FDD is similar to the one described in [8] for reducing a BDD.

As an example, if we apply Algorithm 1 to the FDD in Figure 2, we get the reduced FDD in Figure 5. Note that the FDD in Figure 2 consists of six decision paths, whereas the FDD in Figure 5 consists of three decision paths.

\section{$5 \quad$ FDD Marking}

A firewall rule of the form $F_{1} \in S_{1} \wedge \cdots \wedge F_{d} \in S_{d} \rightarrow\langle$ decision $\rangle$ is simple iff every $S_{i}(1 \leq i \leq d)$ is a nonnegative integer interval. Because most firewalls require simple rules, we want to minimize the number of simple rules generated from an FDD. The number of simple rules generated from a "marked version" of an FDD is less than or equal to the number of simple rules generated from the original FDD. Next, we define a marked FDD.

A marked version $f^{\prime}$ of an FDD $f$ is the same as $f$ except that exactly one outgoing edge of each nonterminal node in $f^{\prime}$ is marked "all". Since the labels of the edges that are marked "all" do not change, the two FDDs $f$ and $f^{\prime}$ have 


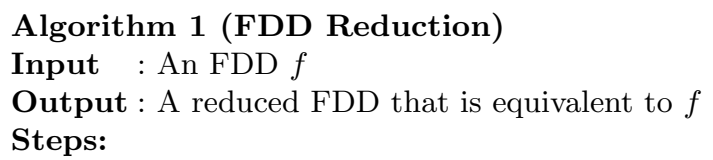

Repeatedly apply the following three reductions to $f$ until none of them can be applied any further.

1. If there is a node $v$ that has only one outgoing edge $e$, assuming $e$ point to node $v^{\prime}$, then remove both node $v$ and edge $e$, and let all the edges that point to $v$ point to $v^{\prime}$.

2. If there are two nodes $v$ and $v^{\prime}$ that are isomorphic, then remove $v^{\prime}$ together with all its outgoing edges, and let all the edges that point to $v^{\prime}$ point to $v$.

3 . If there are two edges $e$ and $e^{\prime}$ that both are between a pair of two nodes, then remove $e^{\prime}$ and change the label of $e$ from $I(e)$ to $I(e) \cup I\left(e^{\prime}\right)$. (Recall that $I(e)$ denotes the label of edge $e$.)

Figure 4. Algorithm 1 (FDD Reduction)

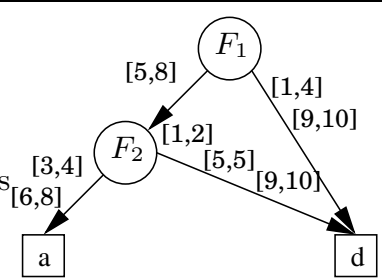

Figure 5. A reduced FDD

the same semantics, i.e., $f$ and $f^{\prime}$ are equivalent. A marked version of an FDD is also called a marked FDD.

Figure 6 shows two marked versions $f^{\prime}$ and $f^{\prime \prime}$ of the FDD in Figure 5. In $f^{\prime}$, the edge labeled $[5,8]$ and the edge labeled $[1,2] \cup[5,5] \cup[9,10]$ are both marked all. In $f^{\prime \prime}$, the edge labeled $[1,4] \cup[9,10]$ and the edge labeled $[1,2] \cup[5,5] \cup[9,10]$ are both marked all.

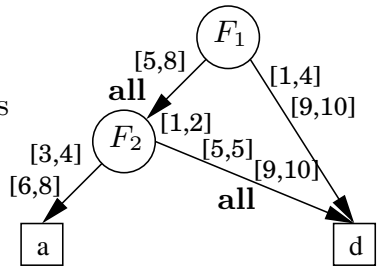

(a) $f^{\prime}$

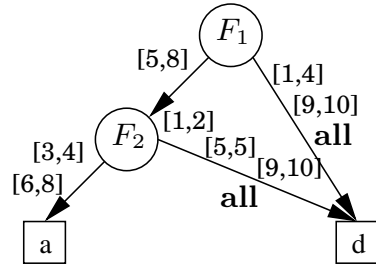

(b) $f^{\prime \prime}$

Figure 6. Two marked FDDs 
The load of a nonempty set of integers $\mathrm{S}$, denoted $\operatorname{load}(S)$, is the minimal number of non-overlapping integer intervals that cover $S$. For example, the load of the set $\{1,2,3,5,8,9,10\}$ is 3 because this set is covered by the three integer intervals $[1,3],[5,5]$ and $[8,10]$, and this set cannot be covered by any two intervals.

The load of an edge $e$ in a marked FDD, denoted load $(e)$, is defined as follows:

$$
\operatorname{load}(e)= \begin{cases}1 & \text { if } e \text { is marked all } \\ \operatorname{load}(I(e)) & \text { otherwise }\end{cases}
$$

The load of a node $v$ in a marked FDD, denoted $\operatorname{load}(v)$, is defined recursively as follows:

$\operatorname{load}(v)= \begin{cases}1 & \text { if } v \text { is terminal } \\ \sum_{i=1}^{k}\left(\operatorname{load}\left(e_{i}\right) \times \operatorname{load}\left(v_{i}\right)\right) & \text { if } v \text { is nonterminal: suppose } v \text { has } k \\ & \text { outgoing edges } e_{1}, \cdots, e_{k}, \text { which point to } \\ & \text { nodes } v_{1}, \cdots, v_{k} \text { respectively }\end{cases}$

The load of a marked FDD $f$, denoted load $(f)$, equals the load of the root of $f$.

Different marked versions of the same FDD may have different loads. Figure 6 shows two marked versions $f^{\prime}$ and $f^{\prime \prime}$ of the same FDD in Figure 5. The load of $f^{\prime}$ is 5 , whereas the load of $f^{\prime \prime}$ is 4 .

As we will see in Section 8, for any two marked versions of the same FDD, the one with the smaller load will generate a smaller number of simple rules. Therefore, we should use the marked version of FDD $f$ that has the minimal load to generate rules.

Algorithm 2 (FDD marking) in Figure 7 takes any FDD and outputs a marked version that has the minimal load.

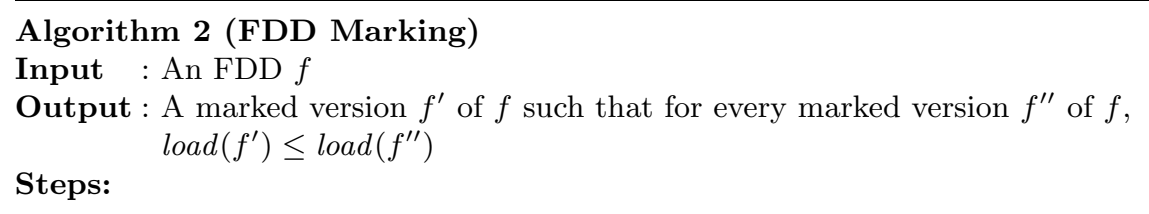

1. Compute the load of each terminal node $v$ in $f$ as follows: $\operatorname{load}(v):=1$

2. while there is a node $v$ whose load has not yet been computed, suppose $v$ has $k$ outgoing edges $e_{1}, \cdots, e_{k}$ and these edges point to nodes $v_{1}, \cdots, v_{k}$ respectively, and the loads of these $k$ nodes have been computed do
(a) Among the $k$ edges $e_{1}, \cdots, e_{k}$, choose an edge $e_{j}$ with the largest value of $\left.\operatorname{load}\left(e_{j}\right)-1\right) \times \operatorname{load}\left(v_{j}\right)$, and mark edge $e_{j}$ with "all".
(b) Compute the load of $v$ as follows: $\operatorname{load}(v):=\sum_{i=1}^{k}\left(\operatorname{load}\left(e_{i}\right) \times \operatorname{load}\left(v_{i}\right)\right)$. end


As an example, if we apply Algorithm 2 to the reduced FDD in Figure 5, we get the marked FDD in Figure 6(b).

The correctness of Algorithm 2 is stated in Theorem 2, whose proof is presented in the appendix.

Theorem 2 The load of an FDD marked by Algorithm 2 (FDD Marking) is minimal.

\section{Firewall Generation}

In this section, we present an algorithm for generating a sequence of rules, which form a firewall, from a marked FDD such that the firewall has the same semantics as the marked FDD. First, we introduce the semantics of a firewall.

A packet $\left(p_{1}, \cdots, p_{d}\right)$ matches a rule $F_{1} \in S_{1} \wedge \cdots \wedge F_{d} \in S_{d} \rightarrow\langle$ decision $\rangle$ iff the condition $p_{1} \in S_{1} \wedge \cdots \wedge p_{d} \in S_{d}$ holds. A firewall consists of a sequence of rules such that for any packet there is at least one rule that the packet matches. A firewall maps every packet to the decision of the first rule that the packet matches. Let $f$ be a firewall of a sequence of rules. The set of all packets accepted by $f$ is denoted f.accept, and the set of all packets discarded by $f$ is denoted f.discard. The next theorem follows from these definitions. Recall that $\Sigma$ denotes the set of all packets over the fields $F_{1}, \cdots, F_{d}$.

Theorem 3 (Theorem of Firewalls) For a firewall $f$ of a sequence of rules,

1. f.accept $\cap$ f.discard $=\emptyset$, and

2. f.accept $\cup$ f.discard $=\Sigma$

Based on Theorem 1 and 3, we now extend the equivalence relations on FDDs to incorporate the firewalls. Given $f$ and $f^{\prime}$, where each is an FDD or a firewall, $f$ and $f^{\prime}$ are equivalent iff they have identical accept sets and identical discard sets, i.e., $f$.accept $=f^{\prime}$.accept and $f$.discard $=f^{\prime}$.discard. This equivalence relation is symmetric, reflexive, and transitive. We use $f \equiv f^{\prime}$ to denote the equivalence relation between $f$ and $f^{\prime}$.

To generate an equivalent firewall from a marked FDD $f$, we basically make a depth-first traversal of $f$ such that for each nonterminal node $v$, the outgoing edge marked "all" of $v$ is traversed after all the other outgoing edges of $v$ have been traversed. Whenever a terminal node is encountered, assuming $\left\langle v_{1} e_{1} \cdots v_{k} e_{k} v_{k+1}\right\rangle$ is the decision path where for every $i(1 \leq i \leq k) e_{i}$ is the most recently traversed outgoing edge of node $v_{i}$, output a rule $r$ as follows:

$$
F_{1} \in S_{1} \wedge \cdots \wedge F_{d} \in S_{d} \rightarrow F\left(v_{k+1}\right)
$$

where

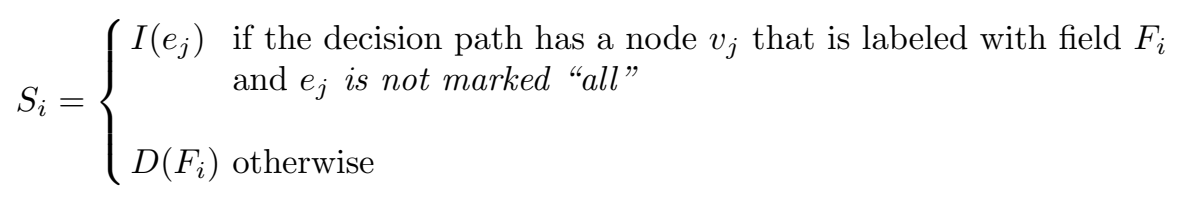

Note that the $i$-th rule output is the $i$-th rule in the firewall generated.

For the above rule $r$, the predicate $F_{1} \in S_{1} \wedge \cdots \wedge F_{d} \in S_{d}$ is called the matching predicate of $r$. 
The rule represented by the path $\left\langle v_{1} e_{1} \cdots v_{k} e_{k} v_{k+1}\right\rangle$ is $F_{1} \in T_{1} \wedge \cdots \wedge F_{d} \in$ $T_{d} \rightarrow F\left(v_{k+1}\right)$, where

$$
T_{i}=\left\{\begin{array}{l}
I\left(e_{j}\right) \text { if the decision path has a node } v_{j} \text { that is labeled with field } F_{i} \\
D\left(F_{i}\right) \text { otherwise }
\end{array}\right.
$$

We call the predicate $F_{1} \in T_{1} \wedge \cdots \wedge F_{d} \in T_{d}$ the resolving predicate of the above rule $r$. Note that if a packet satisfies the resolving predicate of $r, r$ is the first rule that the packet matches in the firewall generated. If a packet satisfies the resolving predicate of rule $r$ in firewall $f$, we say the packet is resolved by $r$ in $f$.

Algorithm 3 (firewall generation) in Figure 8 takes any marked FDD and outputs an equivalent firewall. Recall that the $i$-th rule output by Algorithm 3 is the $i$-th rule in the firewall generated. The correctness of this algorithm follows directly from the semantics of FDDs and firewalls. In Algorithm 3, for every rule generated, we also generate its matching predicate and its resolving predicate. In the next section, we will see that these two predicates associated with each rule play important roles in removing redundant rules.

\footnotetext{
Algorithm 3 (Firewall Generation)

Input : A marked FDD $f$

Output : A firewall that is equivalent to $f$. For each rule $r, r . m p$ and $r . r p$ is computed Steps:
}

Depth-first traverse $f$ such that for each nonterminal node $v$, the outgoing edge marked "all" of $v$ is traversed after all other outgoing edges of $v$ have been traversed. Whenever a terminal node is encountered, assuming $\left\langle v_{1} e_{1} \cdots v_{k} e_{k} v_{k+1}\right\rangle$ is the decision path where each $e_{i}$ is the most recently traversed outgoing edge of node $v_{i}$, output a rule $r$ together with its matching predicate $r . m p$ and its resolving predicate $r . r p$ as follows:

$r$ is the rule $F_{1} \in S_{1} \wedge \cdots \wedge F_{d} \in S_{d} \rightarrow F\left(v_{k+1}\right)$, where

$S_{i}=\left\{\begin{array}{l}I\left(e_{j}\right) \quad \text { if the decision path has a node } v_{j} \text { that is labeled with field } F_{i} \\ \quad \text { and } e_{j} \text { is not marked "all" } \\ D\left(F_{i}\right) \text { otherwise }\end{array}\right.$

r.mp is the predicate of rule $r$.

r.rp is the predicate $F_{1} \in T_{1} \wedge \cdots \wedge F_{d} \in T_{d}$, where

$T_{i}=\left\{\begin{array}{l}I\left(e_{j}\right) \text { if the decision path has a node } v_{j} \text { that is labeled with field } F_{i} \\ D\left(F_{i}\right) \text { otherwise }\end{array}\right.$

Figure 8. Algorithm 3 (Firewall Generation)

As an example, if we apply Algorithm 3 to the marked FDD in Figure 6(b), we get the firewall in Figure 9 .

\section{Firewall Compaction}

Firewalls often have redundant rules. A rule in a firewall is redundant iff removing the rule does not change the semantics of the firewall, i.e., does not change the 


$$
\begin{array}{ll}
r_{1} & =F_{1} \in[5,8] \wedge F_{2} \in[3,4] \cup[6,8] \rightarrow a, \\
r_{1} \cdot m p & =F_{1} \in[5,8] \wedge F_{2} \in[3,4] \cup[6,8] \\
r_{1} \cdot r p & =F_{1} \in[5,8] \wedge F_{2} \in[3,4] \cup[6,8] \\
r_{2} & =F_{1} \in[5,8] \wedge F_{2} \in[1,10] \quad \rightarrow d, \\
r_{2} \cdot m p & =F_{1} \in[5,8] \wedge F_{2} \in[1,10] \\
r_{2} \cdot r p & =\left(F_{1} \in[5,8] \wedge F_{2} \in[1,2] \cup[5,5] \cup[9,10]\right) \\
& =F_{1} \in[1,10] \wedge F_{2} \in[1,10] \quad \rightarrow d, \\
r_{3} & \rightarrow d, \\
r_{3} \cdot m p & =F_{1} \in[1,10] \wedge F_{2} \in[1,10] \\
r_{3} \cdot r p & =F_{1} \in[1,4] \cup[9,10] \wedge F_{2} \in[1,10]
\end{array}
$$

Figure 9. A generated firewall

accept set and the discard set of the firewall. Removing redundant rules from a firewall produces an equivalent firewall but with fewer rules. For example, the rule $r_{2}$ in Figure 9 is redundant. Removing this rule yields an equivalent firewall with two rules, which are shown in Figure 10.

1. $F_{1} \in[5,8] \wedge F_{2} \in[3,4] \cup[6,8] \rightarrow a$,

2. $F_{1} \in[1,10] \wedge F_{2} \in[1,10] \rightarrow d$

Figure 10. A firewall with no redundant rules

In this section, we present an efficient algorithm for discovering redundant rules. Algorithm 4 (firewall compaction) in Figure 11 takes any firewall and outputs an equivalent but more compact firewall.

In Algorithm 4, " $r_{i} . r p$ implies $r_{k} . m p$ " means that for any packet $p$, if $p$ satisfies $r_{i}$.rp, then $p$ satisfies $r_{k} . m p$. Checking whether $r_{i} . r p$ implies $r_{k} . m p$ is simple. Let $r_{i} . r p$ be $F_{1} \in T_{1} \wedge F_{2} \in T_{2} \wedge \cdots \wedge F_{d} \in T_{d}$ and let $r_{k} . m p$ be $F_{1} \in S_{1} \wedge F_{2} \in S_{2} \wedge \cdots \wedge F_{d} \in S_{d}$. Then, $r_{i}$.rp implies $r_{k}$.mp iff for every $j$, where $1 \leq j \leq d$, the condition $T_{j} \subseteq S_{j}$ holds.

Checking whether no packet satisfies both $r_{i} . r p$ and $r_{j} . m p$ is simple. Let $r_{i} . r p$ be $F_{1} \in T_{1} \wedge F_{2} \in T_{2} \wedge \cdots \wedge F_{d} \in T_{d}$ and let $r_{j} . m p$ be $F_{1} \in S_{1} \wedge F_{2} \in S_{2} \wedge \cdots \wedge F_{d} \in$ $S_{d}$. We have $r_{i} . r p \wedge r_{j} . m p=F_{1} \in\left(T_{1} \cap S_{1}\right) \wedge F_{2} \in\left(T_{2} \cap S_{2}\right) \wedge \cdots \wedge F_{d} \in\left(T_{d} \cap S_{d}\right)$. Therefore, no packet satisfies both $r_{i} . r p$ and $r_{j} . m p$ iff there exists $j$, where $1 \leq j \leq d$, such that $T_{j} \cap S_{j}=\emptyset$.

The correctness of Algorithm 4 is stated in Theorem 4, whose proof is presented in the appendix.

Theorem 4 If we apply Algorithm 4 to a firewall $f$ and get the resulting firewall $f^{\prime}$, then $f$ and $f^{\prime}$ are equivalent.

As an example, if we apply Algorithm 4 to the firewall in Figure 9, we get the compact firewall in Figure 10.

Let $n$ be the number of rules in a firewall and $d$ be the number of packet fields that a rule checks, the computational complexity of Algorithm 4 is $O\left(n^{2} * d\right)$. Note 


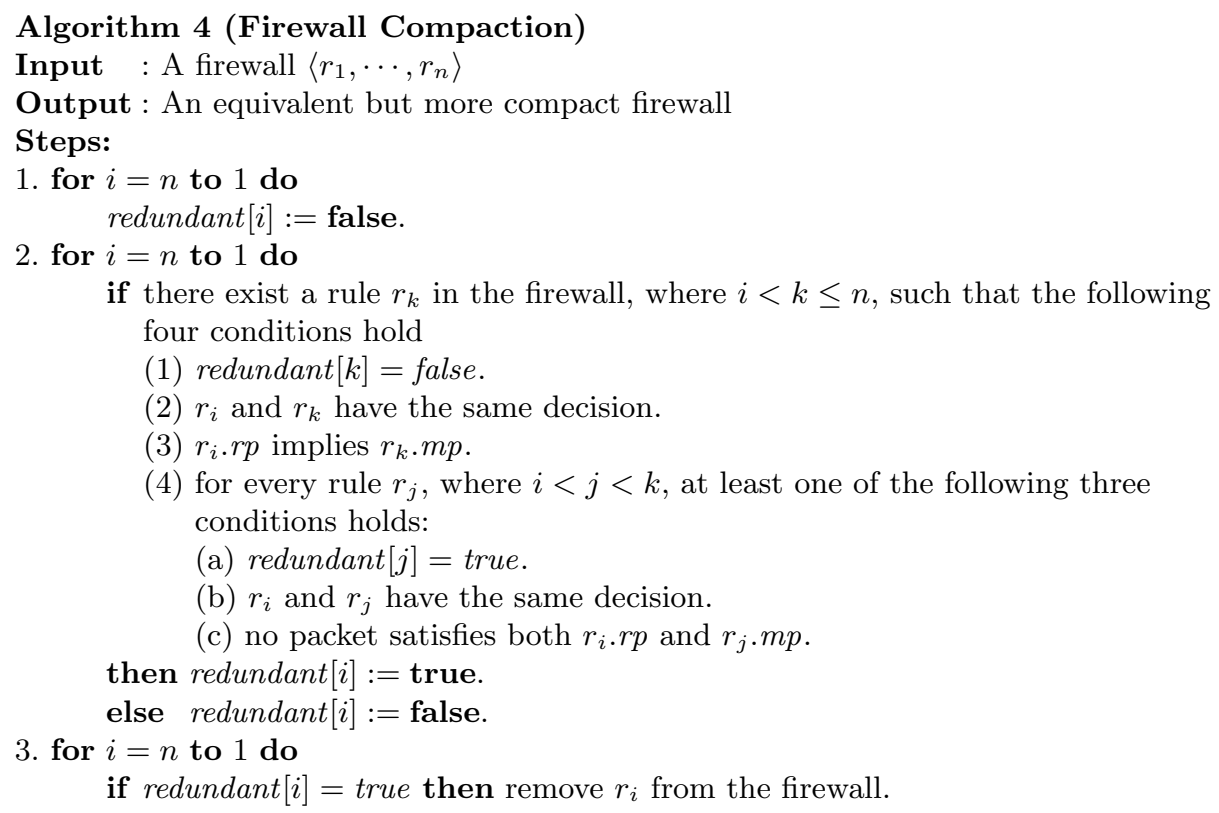

\section{Figure 11. Algorithm 4 (Firewall Compaction)}

that $d$ can be regarded as a constant because $d$ is usually small. Most firewalls checks five packet fields: source IP address, destination IP address, source port number, destination port number, and protocol type.

\section{Firewall Simplification}

Most firewall software, such as Linux's ipchains [2], requires each firewall rule to be simple. A firewall rule of the form $F_{1} \in S_{1} \wedge \cdots \wedge F_{d} \in S_{d} \rightarrow\langle$ decision $\rangle$ is simple iff every $S_{i}(1 \leq i \leq d)$ is an interval of consecutive nonnegative integers. A firewall is simple iff all its rules are simple.

Algorithm 5 (firewall simplification) in Figure 12 takes any firewall and outputs an equivalent firewall in which each rule is simple. The correctness of this algorithm follows directly from the semantics of firewalls.

As an example, if we apply Algorithm 5 to the firewall in Figure 10, we get the firewall in Figure 13.

What we get from Algorithm 5 is a simple firewall. For each rule $F_{1} \in S_{1} \wedge$ $\cdots \wedge F_{i} \in S_{i} \wedge \cdots \wedge F_{d} \in S_{d} \rightarrow\langle$ decision $\rangle, S_{i}$ is an interval of nonnegative integers. Some existing firewall products, such as Linux's ipchains [2], require that $S_{i}$ be represented in a prefix format such as 192.168.0.0/16, where 16 means that the prefix is the first 16 bits of 192.168.0.0 in a binary format. In this paper we stop the level of discussion at simple rules because an integer interval can be converted to multiple prefixes algorithmically. For example, integer interval $[2,8]$ can be converted to 3 prefixes: $001 *, 01 *, 1000$. A $w$-bit integer interval can be converted to at most $2 w-2$ prefixes [14]. 


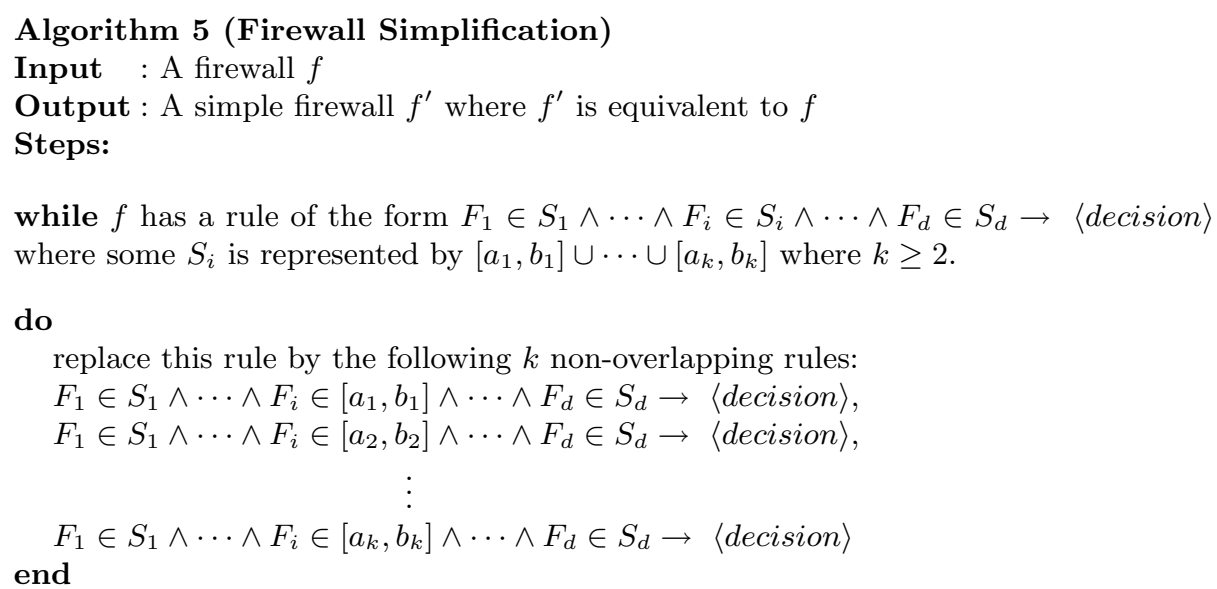

Figure 12. Algorithm 5 (Firewall Simplification)

1. $F_{1} \in[5,8] \wedge F_{2} \in[3,4] \rightarrow a$,

2. $F_{1} \in[5,8] \wedge F_{2} \in[6,8] \rightarrow a$,

3. $F_{1} \in[1,10] \wedge F_{2} \in[1,10] \rightarrow d$,

Figure 13. A simple firewall

\section{Summary of Structured Firewall Design}

In this section, we summarize our firewall design method. Figure 14 shows the five steps of this method.

Our firewall design method starts by a user specifying an FDD $f$. The consistency and completeness properties of $f$ can be verified automatically based on the syntactic requirements of an FDD. After an FDD is specified, it goes through the following five steps, and we get a simple firewall that is equivalent to the FDD. The first step is to apply Algorithm 1 (FDD Reduction) to the user specified FDD. We then get an equivalent but reduced FDD, which has a smaller number of decision paths. The second step is to apply Algorithm 2 (FDD Marking) to the reduced FDD. We then get an equivalent FDD where each nonterminal node has exactly one outgoing edge that is marked all. The third step is to apply Algorithm 3 (FDD Generation) to the marked FDD. We then get an equivalent firewall. The fourth step is to apply Algorithm 4 (Firewall Compaction) to the generated firewall. We then get an equivalent firewall with a smaller number of rules. The fifth step is to apply Algorithm 5 (Firewall Simplification) to this firewall. We then get the final result: a simple firewall that is equivalent to the user specified FDD.

Three of the above five algorithms, namely Algorithm 1 (FDD Reduction), Algorithm 2 (FDD Marking) and Algorithm 4 (Firewall Compaction), are for the purpose of reducing the number of rules in the final simple firewall. Algorithm 1 (FDD Reduction) does so by reducing the number of decision paths in the user specified FDD. Algorithm 2 (FDD Marking) does so by reducing the load of 


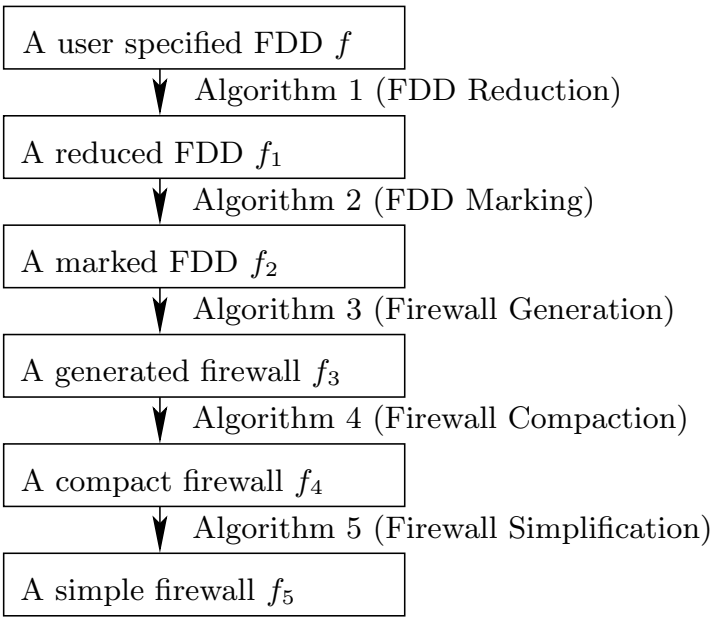

Figure 14. Five steps of our firewall design method ( $f \equiv f_{1} \equiv f_{2} \equiv f_{3} \equiv$ $f_{4} \equiv f_{5}$ )

some edges in the FDD. Algorithm 4 (Firewall Compaction) does so by removing some redundant rules from the generated firewall. These three algorithms could reduce the number of simple rules dramatically. Consider the running example illustrated in Figures 2 through 13. If we directly generate and simplify our firewall from the FDD in Figure 2, ignoring Algorithm 1, 2, and 4, we would have ended up with a simple firewall that has 14 rules. However, with the help of these three algorithms, we end up with a simple firewall that has only 3 rules.

\section{Conclusions}

Our contribution in this paper is two-fold. First, we propose the structured firewall design method that addresses the consistency problem, the completeness problem, and the compactness problem. These three problems are inherent in the current practice of designing a firewall directly as a sequence of (possibly conflicting) rules. Our method starts with a decision diagram that ensures the consistency and completeness properties, and ends up with a sequence of rules that ensures the compactness property. In this process, the user only deals with a firewall decision diagram, which is the formal specification of a firewall. Converting a decision diagram to a compact sequence of rules is automatically carried by a series of five algorithms presented in this paper. Second, we present three optimization techniques, namely FDD reduction, FDD marking, and firewall compaction, for reducing the total number of rules generated from a firewall decision diagram.

In this paper, for ease of presentation, we assume that a firewall maps every packet to one of two decisions: accept or discard. Most firewall software supports more than two decisions such as accept, accept-and-log, discard, and discard-and- 
log. Our firewall design method can be straightforwardly extended to support more than two decisions.

The design method discussed in this paper is not limited to just firewalls. Rather, the techniques and algorithms presented in this paper are extensible to other rule-based systems such as general packet classifiers and IPsec rules.

\section{References}

1. High level firewall language, http://www.hlfl.org/.

2. ipchains, http://www.tldp.org/howto/ipchains-howto.html.

3. E. Al-Shaer and H. Hamed. Discovery of policy anomalies in distributed firewalls. In IEEE INFOCOM'04, pages 2605-2616, March 2004.

4. F. Baboescu and G. Varghese. Fast and scalable conflict detection for packet classifiers. In Proceedings of the 10th IEEE International Conference on Network Protocols, 2002.

5. Y. Bartal, A. J. Mayer, K. Nissim, and A. Wool. Firmato: A novel firewall management toolkit. In Proceeding of the IEEE Symposium on Security and Privacy, pages 17-31, 1999.

6. Y. Bartal, A. J. Mayer, K. Nissim, and A. Wool. Firmato: A novel firewall management toolkit. Technical Report EES2003-1, Dept. of Electrical Engineering Systems, Tel Aviv University, 2003.

7. A. Begel, S. McCanne, and S. L. Graham. BPF+: Exploiting global data-flow optimization in a generalized packet filter architecture. In Proceedings of $A C M$ SIGCOMM '99, 1999.

8. R. E. Bryant. Graph-based algorithms for boolean function manipulation. IEEE Trans. on Computers, 35(8):677-691, 1986.

9. CERT. Test the firewall system. http://www.cert.org/

security-improvement/practices/p060.html.

10. E. W. Dijkstra. Goto statement considered harmful. Communications of the ACM, 11(3):147-148, March 1968.

11. D. Eppstein and S. Muthukrishnan. Internet packet filter management and rectangle geometry. In Symp. on Discrete Algorithms, pages 827-835, 2001.

12. M. Frantzen, F. Kerschbaum, E. Schultz, and S. Fahmy. A framework for understanding vulnerabilities in firewalls using a dataflow model of firewall internals. Computers and Security, 20(3):263-270, 2001.

13. M. G. Gouda and A. X. Liu. A model of stateful firewalls and its properties. In Proceedings of the IEEE International Conference on Dependable Systems and Networks (DSN-05), pages 320-327, June 2005.

14. P. Gupta and N. McKeown. Algorithms for packet classification. IEEE Network, $15(2): 24-32,2001$.

15. J. D. Guttman. Filtering postures: Local enforcement for global policies. In Proceedings of IEEE Symp. on Security and Privacy, pages 120-129, 1997.

16. A. Hari, S. Suri, and G. M. Parulkar. Detecting and resolving packet filter conflicts. In Proceedings of IEEE INFOCOM, pages 1203-1212, 2000.

17. S. Hazelhurst. Algorithms for analyzing firewall and router access lists. Technical Report TR-Wits-CS-1999-5, Department of Computer Science, University of the Witwatersrand, South Africa, July 1999.

18. S. Hazelhurst, A. Attar, and R. Sinnappan. Algorithms for improving the dependability of firewall and filter rule lists. In Proceedings of the Workshop on Dependability of IP Applications, Platforms and Networks, 2000.

19. S. Kamara, S. Fahmy, E. Schultz, F. Kerschbaum, and M. Frantzen. Analysis of vulnerabilities in internet firewalls. Computers and Security, 22(3):214-232, 2003.

20. A. X. Liu, M. G. Gouda, H. H. Ma, and A. H. Ngu. Firewall queries. In Proceedings of the 8th International Conference on Principles of Distributed Systems, LNCS 3544, T. Higashino Ed., Springer-Verlag, pages 124-139, December 2004. 
21. A. Mayer, A. Wool, and E. Ziskind. Fang: A firewall analysis engine. In Proceedings of IEEE Symp. on Security and Privacy, pages 177-187, 2000.

22. J. D. Moffett and M. S. Sloman. Policy conflict analysis in distributed system management. Journal of Organizational Computing, 4(1):1-22, 1994.

23. G. Patz, M. Condell, R. Krishnan, and L. Sanchez. Multidimensional security policy management for dynamic coalitions. In Proceedings of the DARPA Information Survivability Conference and Exposition (DISCEX II), June 2001.

24. J. Quinlan. Induction of decision trees. Machine Learning, 1(1):81-106, 1986.

25. K. Strehl and L. Thiele. Interval diagrams for efficient symbolic verification of process networks. IEEE Trans. on Computer-Aided Design of Integrated Circuits and Systems, 19(8):939-956, 2000.

26. A. Wool. Architecting the lumeta firewall analyzer. In Proceedings of the 10th USENIX Security Symposium, pages 85-97, August 2001.

27. A. Wool. A quantitative study of firewall configuration errors. IEEE Computer, $37(6): 62-67,2004$.

\section{Appendix}

\section{A Proof of Algorithm 2}

Consider an FDD $f$. Let $f^{\prime}$ be the version marked by algorithm 2 , and let $f^{\prime \prime}$ be an arbitrary marked version. Next we prove that $\operatorname{load}\left(f^{\prime}\right) \leq \operatorname{load}\left(f^{\prime \prime}\right)$.

Consider a node $v$, which has $k$ outgoing edges $e_{1}, e_{2}, \cdots, e_{k}$ and these edges point to $v_{1}, v_{2}, \cdots, v_{k}$ respectively, such that the loads of $v_{1}, v_{2}, \cdots, v_{k}$ in $f^{\prime}$ is the same as those in $f^{\prime \prime}$. Clearly such node $v$ exists because the load of any terminal node is constant 1 .

Let $e_{i}$ be the edge marked $A L L$ in $f^{\prime}$ and $e_{j}$ be the edge marked $A L L$ in $f^{\prime \prime}$. Suppose $i \neq j$. We use $\operatorname{load}^{\prime}(v)$ to denote the load of node $v$ in $f^{\prime}$ and $\operatorname{load}^{\prime \prime}(v)$ to denote the load of node $v$ in $f^{\prime \prime}$. We then have

$$
\begin{aligned}
& \operatorname{load}^{\prime}(v)=\sum_{t=1}^{i-1}\left(\operatorname{load}\left(e_{t}\right) \times \operatorname{load}\left(v_{t}\right)\right)+\operatorname{load}\left(v_{i}\right)+\sum_{t=i+1}^{k}\left(\operatorname{load}\left(e_{t}\right) \times \operatorname{load}\left(v_{t}\right)\right) \\
& \operatorname{load}^{\prime \prime}(v)=\sum_{t=1}^{j-1}\left(\operatorname{load}\left(e_{t}\right) \times \operatorname{load}\left(v_{t}\right)\right)+\operatorname{load}\left(v_{j}\right)+\sum_{t=j+1}^{k}\left(\operatorname{load}\left(e_{t}\right) \times \operatorname{load}\left(v_{t}\right)\right) \\
& \operatorname{load}^{\prime}(v)-\operatorname{load}^{\prime \prime}(v)=\left(\operatorname{load}\left(e_{j}\right)-1\right) \times \operatorname{load}\left(v_{j}\right)-\left(\operatorname{load}\left(e_{i}\right)-1\right) \times \operatorname{load}\left(v_{i}\right)
\end{aligned}
$$

According to Algorithm 2, $\left(\operatorname{load}\left(e_{j}\right)-1\right) \times \operatorname{load}\left(v_{j}\right) \leq\left(\operatorname{load}\left(e_{i}\right)-1\right) \times \operatorname{load}\left(v_{i}\right)$. So, $\operatorname{load}^{\prime}(v) \leq \operatorname{load}^{\prime \prime}(v)$.

Apply the above argument to any node $v$ in $f$, we have $\operatorname{load}^{\prime}(v) \leq \operatorname{load}^{\prime \prime}(v)$. So, the load of an FDD marked by Algorithm 2 is minimal.

\section{B Proof of Theorem 4}

Suppose for the rule $r_{i}$ in firewall $\left\langle r_{1}, \cdots, r_{n}\right\rangle$, there exist a rule $r_{k}$ in this firewall, where $i<k \leq n$, such that the following four conditions hold:

1. redundant $[k]=$ false.

2. $r_{i}$ and $r_{k}$ have the same decision.

3. $r_{i}$.rp implies $r_{k}$. mp .

4. for every rule $r_{j}$, where $i<j<k$, at least one of the following three conditions holds:

(a) redundant $[j]=$ true.

(b) $r_{i}$ and $r_{j}$ have the same decision.

(c) no packet satisfies both $r_{i} . r p$ and $r_{j} . m p$. 
If we remove rule $r_{i}$ from firewall $\left\langle r_{1}, \cdots, r_{n}\right\rangle$, the packets whose decision may be affected are the packets that are resolved by $r_{i}$ in $\left\langle r_{1}, \cdots, r_{n}\right\rangle$, i.e., the packets that satisfy $r_{i} . r p$. Let $S$ be the set of all the packets that satisfy $r_{i} . r p$. Because $r_{i} . r p$ implies $r_{k} . m p$ and redundant $[k]=$ false, if we remove rule $r_{i}$, the packets in $S$ will be resolved by the rules from $r_{i+1}$ to $r_{k}$ in $\left\langle r_{1}, \cdots, r_{i-1}, r_{i+1}, \cdots, r_{n}\right\rangle$. Consider a rule $r_{j}$ where $i<j<k$. If redundant $[j]=$ true, we assume $r_{j}$ has been removed; therefore, rule $r_{j}$ does not affect the decision of any packet in $S$. If the two rules $r_{i}$ and $r_{j}$ have the same decision, then rule $r_{j}$ does not affect the decision of any packet in $S$. If no packet satisfies both $r_{i} . r p$ and $r_{j} . m p$, then any packet in $S$ does not match rule $r_{j}$; therefore, rule $r_{j}$ does not affect the decision of any packet in $S$. Note that $r_{i}$ and $r_{k}$ have the same decision. Therefore, for any packet $p$ in $S$, the decision that the firewall $\left\langle r_{1}, \cdots, r_{i-1}, r_{i+1}, \cdots, r_{n}\right\rangle$ makes for $p$ is the same as the decision that the firewall $\left\langle r_{1}, \cdots, r_{i-1}, r_{i}, r_{i+1}, \cdots, r_{n}\right\rangle$ makes for $p$. So rule $r_{i}$ is redundant.

Suppose we apply Algorithm 4 to a firewall $f$. Since any rule removed by Algorithm 4 is redundant, the resulting firewall $f^{\prime}$ is equivalent to the original firewall $f$. 